CORRIGENDUM

doi:10.1038/nature14555

\title{
Corrigendum: Pan-viral specificity of IFN-induced genes reveals new roles for cGAS in innate immunity
}

John W. Schoggins, Donna A. MacDuff, Naoko Imanaka, Maria D. Gainey, Bimmi Shrestha, Jennifer L. Eitson, Katrina B. Mar, R. Blake Richardson, Alexander V. Ratushny, Vladimir Litvak, Rea Dabelic, Balaji Manicassamy, John D. Aitchison, Alan Aderem, Richard M. Elliott, Adolfo García-Sastre, Vincent Racaniello, Eric J. Snijder, Wayne M. Yokoyama, Michael S. Diamond, Herbert W. Virgin \& Charles M. Rice

Nature 505, 691-695 (2014); doi:10.1038/nature12862

In this Letter, we carried out bioinformatic analyses on interferonstimulated gene screening data sets for multiple viruses, including a data set for West Nile virus (WNV) (Supplementary Table 8 in ref. 1). We recently discovered that the WNV-GFP stock used in our 2011 study $^{1}$ was actually Venezuelan equine encephalitis virus (VEEV-GFP). The error has been tracked to a technical mistake made during the virus production process. Several data sets in this Letter are therefore mislabelled. In Fig. 3a and in all panels of Extended Data Fig. 2a, 'WNV' should be 'VEEV'. The original figure legends remain valid, as do all the other figures in this Letter. One conclusion of the Letter highlighted differences in interferon-stimulated gene specificity between positive-sense and negative-sense RNA viruses. Since VEEV and WNV are both positive-sense, the stated conclusions remain unchanged; all other results and conclusions are also unchanged.

1. Schoggins, J. W. et al. A diverse range of gene products are effectors of the type I interferon antiviral response. Nature 472, 481-485 (2011); corrigendum Nature http://dx.doi.org/10.1038/nature14554 (2015). 